\title{
Adding Chemotherapy After Neoadjuvant Chemoradiation in Locally Advanced Rectal Cancer; Promising or not?
}

\author{
Mehmet A.N SENDUR ${ }^{1}$, Burak BILGIN ${ }^{1}$, Sercan AKSOY ${ }^{2}$, Bülent AKINCI ${ }^{1}$, \\ Nuriye OZDEMIR ${ }^{1}$, Didem S. DEDE ${ }^{1}$, Bülent YALCIN ${ }^{1}$, Nurullah ZENGIN ${ }^{1}$ \\ ${ }^{1}$ Yıldırım Beyazıt University, Faculty of Medicine, Department of Medical Oncology \\ ${ }^{2}$ Hacettepe University Cancer Institute, Department of Medical Oncology, Ankara, TURKEY
}

To the editor,

Neoadjuvant or adjuvant treatment of stage II or III usually includes locoregional teratment due to the high risk of locoregional recurrence. According to prospective, randomized phase III German Rectal Study Group (CAO/ARA/AIO-94) trial, preoperative chemooradiotherapy significantly improved local control with reduced toxicity compared to postoperative chemoradiotherapy in clinical stage T3 or T4 or node-positive rectal cancer patients. ${ }^{1}$ In a study of $\mathrm{T} 3$ or $\mathrm{T} 4$ rectal cancer patients pre-operative concurrent chemoradiotherapy with 5-Fluorouracil and leucovorin combination significantly increased pathological complete response (pCR) rate and significantly decreased local recurrence rate compared to radiotherapy alone despite no overall survival benefit. ${ }^{2}$ In a systematic review of 5 randomized trials, it was reported that preoperative chemoradiotherapy significantly increased pCR rate and improved local control in resectable stage II and III rectal cancer, but did not improve disease free or overall survival compared to preoperative radiotherapy alone. ${ }^{3}$

To increase response rate and overall survival several large randomized phase III trials investigated the efficacy of adding oxaliplatin to concurrent chemoradiotherapy regimen. In NSABP-R04, STAR 01 and ACCORD12/405-prodige 2 trials, addition of oxaliplatin did not improve pCR rate, also increased grade 3-4 toxicity. On the other hands, recently published in a phase III German CAO/ARO/AIO-04 trial, pCR and the primary endpoint 3-year disease free survival (DFS) significantly improved with adding oxaliplatin to preoperative concurrent chemoradiotherapy and postsurgical infusional fluorouracil. ${ }^{4}$

Several small trials investigated the efficacy of induction chemotherapy before chemoradiotherapy. In a small phase II randomized study of Grupo cancer de recto 3 (GCR-3), the primary endpoint pCR did not differ between chemoradiotherapy arm alone or with induction chemotherapy in patients with locally advanced rectal cancer. ${ }^{5}$ Similarly in another small phase II randomized trial, induction chemotherapy with oxaliplatin did not improve pCR rate compared to chemoradiotherapy alone in patients with locally advanced rectal cancer. ${ }^{6}$

Neoadjuvant short-course radiotherapy or chemoradiation are usually performed to increase tumor resection, induce tumor regression and to reduce the risk of local recurrence. In the Lancet Oncology, recently, Garcia-Aguilar and colleagues reported the role of adding mFOLFOX6 upto 6 cycles after chemoradiation. In this trial, patients non-randomly assigned to neoadjuvant chemoradiation arm or chemoradiation plus 2 or 4 or 6 cycles mFOLFOX6 (Fluoro-uracil, Oxaliplatin, leucovorin). The primary endpoint was $\mathrm{pCR}$. The $\mathrm{pCR}$ rates increased significantly from $18 \%$ upto $38 \%$ in locally advanced rectal cancer with the adding cycles increases upto 6 cycles. Pathological complete response rates were 18\%, 25\%, 30\% and $38 \%$ in patients chemoradiotherapy

UHOD Number: 3 Volume: 27 Year: 2017 
alone, with 2 cycles, 4 cycles or 6 cycles, respectively. Also with adding mFOLFOX6, the mean interval from completion of chemoradiotherapy to surgery was also significantly increased from 8.5 weeks upto 19.3 weeks $(\mathrm{p}<0.0001)$. However, the interpretation of these findings needs several considerations. $^{7}$

Before to comment that adding chemotherapy after chemoradiotherapy improve survival in locally advanced rectal cancer diagnosis, we should mention some confounding factors that affect pCR. In a pooled analysis of patients data including 3105 patients, it was shown that there was a significant clinical association with $\mathrm{pCR}$ rates and clinical T-stage of the disease. The pCR rates decreased from $58 \%$ to $12 \%$ with rising clinical T1-stage to T4 stage. ${ }^{8}$ In the preliminary results of this trial, Garcia-Aguilar et al reported that pCR rates did not improve significantly with adding two cycles mFOLFOX6 after chemoradiotherapy (18\% vs $25 \%) .^{7}$ Although, the ypT0 stage significantly increased from $23 \%$ to $31 \%$ whereas ypN0 stage did not differ with adding 2 cycles chemotherapy $(25 \%$ vs $25 \%$ ). However, there was unequal nonsignificant clinical nodal distribution between treatment groups in the Garcia-Aguilar et al study which can also effect the pCR rates. In addition to, there are no available data about the effect of clinical $\mathrm{T}$ or $\mathrm{N}$ stage on pCR rates Garcia-Aguilar et al study. ${ }^{7}$

In a phase II ADORE (ADjuvant Oxaliplatin in REctal cancer) trial, patients with postoperative pathological stage II (ypT3-4N0) or III (ypT any N1-2) rectal cancer randomly assigned to eight cycles of FOLFOX or four cycles of fluorouracil and leucovorin. ${ }^{9,10}$ Despite 3-year DFS rate increased significantly from $62.9 \%$ to $71.6 \%$, the addition oxaliplatin did not increase DFS significantly in pathologic stage II patients. Interim assesments were done with proctoscopic examination during mFOLFOX6 treatment and the total mesorectal excision was done if the patient had stable or progressive disease but if patients had partial response or pCR the mFOLFOX6 chemotherapy was continued. Although $\mathrm{pCR}$ rates significantly increased in this study, the effect of addition the mFOLFOX6 regimen can not be estimated correctly in patients with partial response or $\mathrm{pCR}$ in the interim assesment. As authors stated in the discussion sec- tion, time from the end of chemoradiotherapy to surgery significantly increased pCR rates. In addition to, the interim results of Stockholm 3 trial also showed that $\mathrm{pCR}$ rates significantly increased from $<1 \%$ to $13 \%$ in patients receving 5-day short course radiotherapy in immediate surgery and 4-8 weeks delays group, respectively. ${ }^{10}$ Although, the authors stated that the time from chemoradiation to surgery can contribute to $\mathrm{pCR}$ rates remains speculative than the administration of mFOLFOX6, we can not give up this idea after the equalization time interval from completion of chemoradiation to surgery between treatment groups. In conclusion, the unequal distribution clinical nodal stage and time interval from the end of chemoradiation treatment between treatment groups have had a bias effect on pCR outcome regarding the adding mFOLFOX6 regimen.

\section{REFERENCES}

1. Sauer R, Becker H, Hohenberger W, et al. Preoperative versus postoperative chemoradiotherapy for rectal cancer. N Engl J Med 351: 1731-1740, 2004.

2. Gerard JP, Conroy T, Bonnetain F, et al. Preoperative radiotherapy with or without concurrent fluorouracil and leucovorin in T3-4 rectal cancers: results of FFCD 9203. J Clin Oncol 24: 4620-4625.

3. De Caluwe L, Van Nieuwenhove Y, Ceelen WP. Preoperative chemoradiation versus radiation alone for stage II and III resectable rectal cancer. Cochrane Database Syst Rev 2013; 2: CD006041. doi: 10.1002/14651858.CD006041.pub3.

4. Rodel C, Graeven U, Fietkau R, et al. Oxaliplatin added to fluorouracil-based preoperative chemoradiotherapy and postoperative chemotherapy of locally advanced rectal cancer (the German CAO/ARO/AIO-04 study): final results of the multicentre, open-label, randomised, phase 3 trial. Lancet Oncol 16: 979-989, 2015.

5. Fernandez-Martos C, Pericay C, Aparicio J, et al. Phase II, randomized study of concomitant chemoradiotherapy followed by surgery and adjuvant capecitabine plus oxaliplatin (CAPOX) compared with induction CAPOX followed by concomitant chemoradiotherapy and surgery in magnetic resonance imaging-defined, locally advanced rectal cancer: Grupo cancer de recto 3 study. J Clin Oncol 28: 859-65, 2010.

6. Marechal R, Vos B, Polus M, et al. Short course chemotherapy followed by concomitant chemoradiotherapy and surgery in locally advanced rectal cancer: a randomized multicentric phase II study. Ann Oncol 23: 1525-1530, 2012. 
7. Garcia-Aguilar J, Chow OS, Smith DD, et al. Effect of adding mFOLFOX6 after neoadjuvant chemoradiation in locally advanced rectal cancer: a multicentre, phase 2 trial. Lancet Oncol 16: 957-966, 2015.

8. Maas M, Nelemans PJ, Valentini V, et al. Long-term outcome in patients with a pathological complete response after chemoradiation for rectal cancer: a pooled analysis of individual patient data. Lancet Oncol 11: 835-844, 2010.

9. Hong YS, Nam BH, Kim KP, et al. Oxaliplatin, fluorouracil, and leucovorin versus fluorouracil and leucovorin as adjuvant chemotherapy for locally advanced rectal cancer after preoperative chemoradiotherapy (ADORE): an open-label, multicentre, phase 2, randomised controlled trial. Lancet Oncol 15: 1245-1253, 2014.

10. Pettersson D, Cedermark B, Holm T, et al. Interim analysis of the Stockholm III trial of preoperative radiotherapy regimens for rectal cancer. Br J Surg 97: 580-587, 2010.

\section{Correspondence:}

Dr. Mehmet Ali Nahit ŞENDUR

Ankara Yildirim Beyazıt Üniversitesi, Tip Fakultesi

Tibbi Onkoloji Anabilim Dali

06800, Bilkent, ANKARA / TURKEY

ANKARA / TURKEY

Tel: (+90-312) 2912525

e-mail: masendur@yahoo.com.tr 